\title{
Application of Fractional Sensor Fusion Algorithms for Inertial MEMS Sensing
}

\author{
M. Romanovas ${ }^{1}$, L. Klingbeil ${ }^{2}$, M. Traechtler ${ }^{3}$ and \\ Y. Manoli ${ }^{4}$ \\ 1,2,3,4 HSG-IMIT Institute of Micromachining and Information Technology \\ Wilhelm-Schickard-Straße 10, D78052, Villingen-Schwenningen, Germany \\ ${ }^{1,4}$ Chair of Microelectronics, Department of Microsystems Engineering \\ IMTEK), University of Freiburg \\ Georges-Köhler-Allee 101, D79110, Freiburg, Germany \\ E-mail(corresp.): michailas.romanovas@hsg-imit.de; \\ E-mail: lasse.klingbeil@hsg-imit.de;martin.traechtler@hsg-imit.de \\ E-mail: manoli@imtek.de
}

Received September 30, 2008; revised January 19, 2009; published online April 30, 2009

\begin{abstract}
The work presents an extension of the conventional Kalman filtering concept for systems of fractional order (FOS). Modifications are introduced using the Grünwald-Letnikov (GL) definition of the fractional derivative (FD) and corresponding truncation of the history length. Two versions of the fractional Kalman filter (FKF) are shown, where the FD is calculated directly or by augmenting the state vector with the estimate of the FD. The filters are compared to conventional integer order (IO) Position (P-KF) and Position-Velocity (PV-KF) Kalman filters as well as to an adaptive Interacting Multiple-Model Kalman Filter (IMM-KF). The performance of the filters is assessed based on a hand and a head motion data set. The feasibility of the given approach is shown.
\end{abstract}

Key words: Kalman filter, fractional-order system,fractional filtering, sensor fusion, Grünwald-Letnikov derivative.

\section{Introduction}

Progress in developing low-cost MEMS (Microelectromechanical Systems) sensors and significant increase of available computational power of embedded systems stimulated research on developing algorithms for combining (fusing) sensors with complementary properties within a single system. The fusion is typically performed via an optimal or suboptimal estimator, where Kalman filters or its modifications are usually employed. The fusion is based on a motion model in the prediction step and the measurements of different modalities during the correction stage. The final performance of such a system is better 
than that based on a single sensor type. The work extends the conventional sensor fusion schemes for FOS starting with a basic linear Kalman Filters.

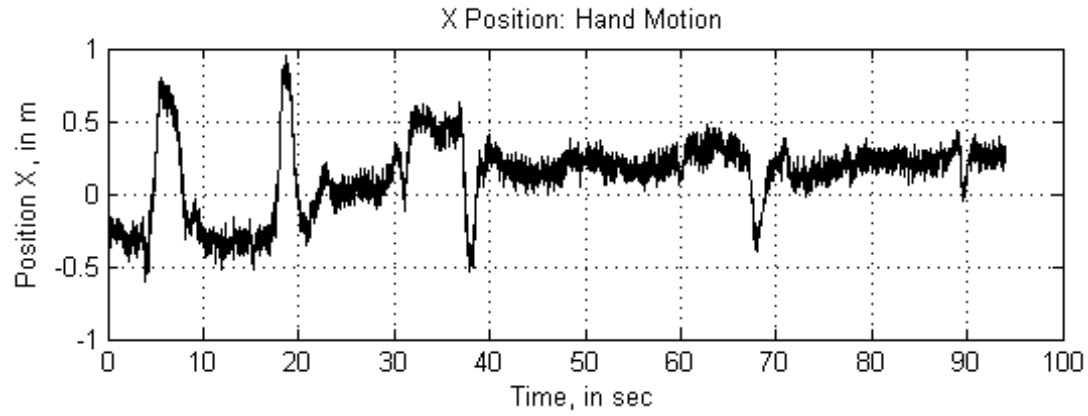

a)

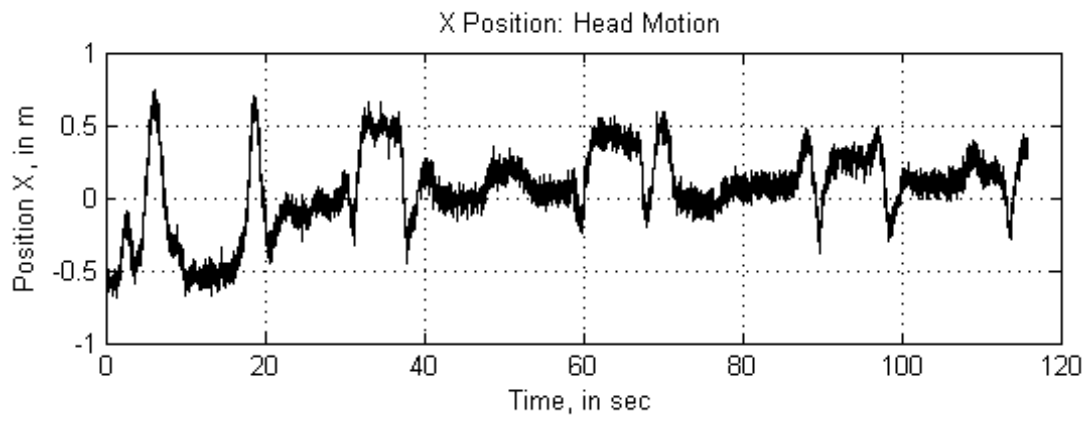

b)

Figure 1. Example of real motion measurements with added Gaussian noise: X component of position measurements a) for hand and b) head motion.

Although the number of applications where fractional calculus (FC) is used, has significantly increased in the last years [3], it still lacks wide acceptance in scientific and engineering community [13]. It could be explained not only by the absence of intuitive geometrical and physical interpretation as there is for some IO operators $[5,13]$, but as well by an existence of several alternative definitions $[5,10]$, which impose extra work in comprehensing and applying FC. Recent achievements in understanding and interpreting FC operators $[5,13,14]$ and development of efficient tools for FOS [7, 12] allowed to apply FC for the processes which are better described with fractional order (FO) rather than IO models. One could refer [6, 12] for formal definitions of FC with results on recent unifying approach in [10]. Extension of the classical system theory for both continuous-time and discrete-time fractional linear system is provided in $[8,9]$ with a tutorial on fractional order signal processing techniques in [2]. The role of FC in soft matter physics, theory of complex materials and viscoelastic behavior, its ability to include effects with non-conservative forces and power-law phenomena propose an idea to capture the complexity of 


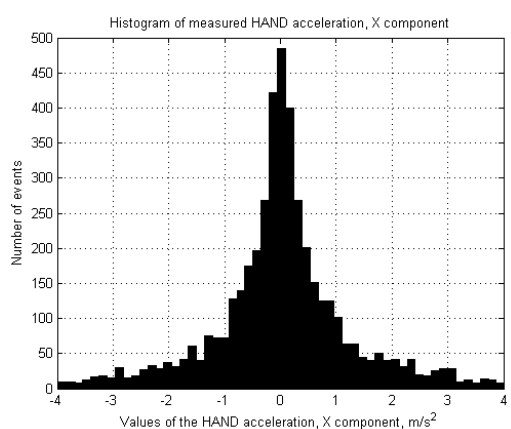

a)

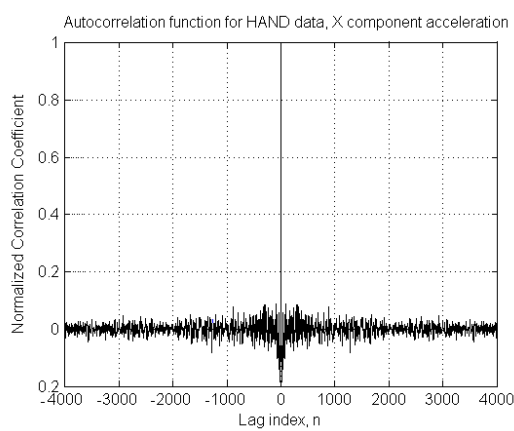

b)

Figure 2. a) Histogram of measured acceleration for ideal hand motion $X$ component and b) autocorrelation function. Measurements performed in a global coordinate frame. Similar results hold for increments of measured accelerations (not shown).

human dynamics (see Fig. 1) by proper extension of filtering schemes with FC operators. The idea is supported by the evidences [3] of $s^{k}$ dynamics in muscles and joint tissues throughout human musculoskeletal system. The Kalman Filter assumes the process being driven by Gaussian noise. Recent work [16] suggested the acceleration of the human limbs to be better represented with Fractional Brownian Motion. Indeed, for our test signals in global frame we have checked both accelerations and their increments of being normally distributed using the Jarque-Bera normality test, where the hypothesis was rejected with $\alpha=0.05$. Thus the assumption about Gaussian noise is violated. Tails in measured distribution and significant values of autocorrelation function for non-zero sample lag can be seen in Fig 2. Thus the concept of FD is introduced as a model for some of the effects described above.

The rest of the paper is organized as follows: after revision of the basic concepts of FC and Kalman filtering in Section 2, we present construction of linear FKF in Section 3 and compare their performance to conventional IO schemes for tracking real human limb motion in Section 4 with the concluding remarks at the end of the paper.

\section{Theory}

The continuous differential and integral operators can be generalized into one operator ${ }_{a} D_{t}^{\alpha}$ as [2]:

$$
{ }_{a} D_{t}^{\alpha}= \begin{cases}\frac{d^{\alpha}}{d t^{\alpha}} & \Re(\alpha)>0 \\ 1 & \Re(\alpha)=0 \\ \int_{a}^{t}(d \tau)^{-\alpha} & \Re(\alpha)<0\end{cases}
$$


where $a$ and $t$ are the limits of operation with alternative definitions of FD in [11]. The choice of the GL definition is caused by its suitability for data which are not necessarily defined via known functional relation and by its straightforward computational scheme for a causal signal:

$$
\begin{aligned}
{ }_{a} D_{t}^{\alpha}[f(t)] & =\lim _{h \rightarrow 0} \frac{1}{h^{\alpha}} \sum_{k=0}^{\left\lfloor\frac{t-a}{h}\right\rfloor}(-1)^{k}\left(\begin{array}{l}
\alpha \\
k
\end{array}\right) f(t-k h) \\
& =\lim _{h \rightarrow 0} \frac{1}{h^{\alpha}} \sum_{k=0}^{\left\lfloor\frac{t-a}{h}\right\rfloor}(-1)^{k} \frac{\Gamma(\alpha+1)}{k ! \Gamma(\alpha-k+1)} f(t-k h) .
\end{aligned}
$$

Here $\lfloor x\rfloor$ means the integer part of $x$ and $\Gamma(x)$ is Euler's gamma function. This can be considered as a generalization of the conventional integer $n$-th derivative operator with backward finite difference. Although the IO derivative needs a finite number of terms and therefore is a local operator, the FD requires an infinite number of terms and differently from IO derivatives has a memory of all the past events.

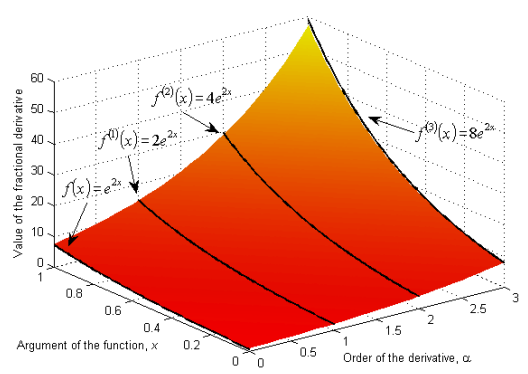

a)

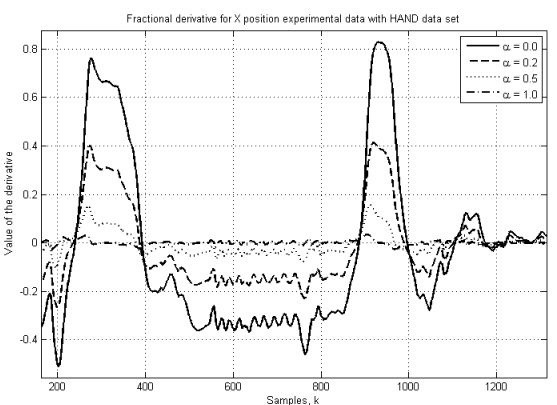

b)

Figure 3. a) GL derivative used to calculate FO and IO derivatives of $f(x)=e^{2 x}(h=0.01)$. b) Example of FD for the real measured $\mathrm{X}$ axis displacement (HAND data set, $T_{s}=1 \mathrm{sec}$ )

The basic limitation of the GL definition is the summation which results in a limited accuracy of the calculations. For numerical calculation of FD we can use the following discrete approximation derived from the GL definition:

$$
{ }_{(t-L)} D_{t}^{\alpha}[f(t)] \approx \frac{1}{h^{\alpha}} \sum_{k=0}^{N(t)} b_{k} f(t-k h), \quad N(t)=\min \left\{\left\lfloor\frac{t}{h}\right\rfloor,\left\lfloor\frac{L}{h}\right\rfloor\right\}
$$

where $L$ is the length of the memory, $h$ is the step size of the calculation and $N$ is the number of coefficients used in the approximation. The binomial coefficients $b_{k}$ are calculated as follows:

$$
b_{0}^{(\alpha)}=1, b_{k}^{(\alpha)}=\left(1-\frac{1+\alpha}{k}\right) b_{k-1}^{(\alpha)} .
$$


GL definition can be directly applied for numerical evaluation of FD in the case of analytically defined functions (Fig. 3a) as well as real measured signals (Fig. $3 \mathrm{~b})$. For detailed treatment of $\mathrm{FC}$ one can refer to $[7,12,15]$.

Kalman filter ensures an optimal solution under a number of assumptions about system noise and dynamics and it became a major tool for solving linear filtering problems due to its efficient recursive scheme (Fig. 4).

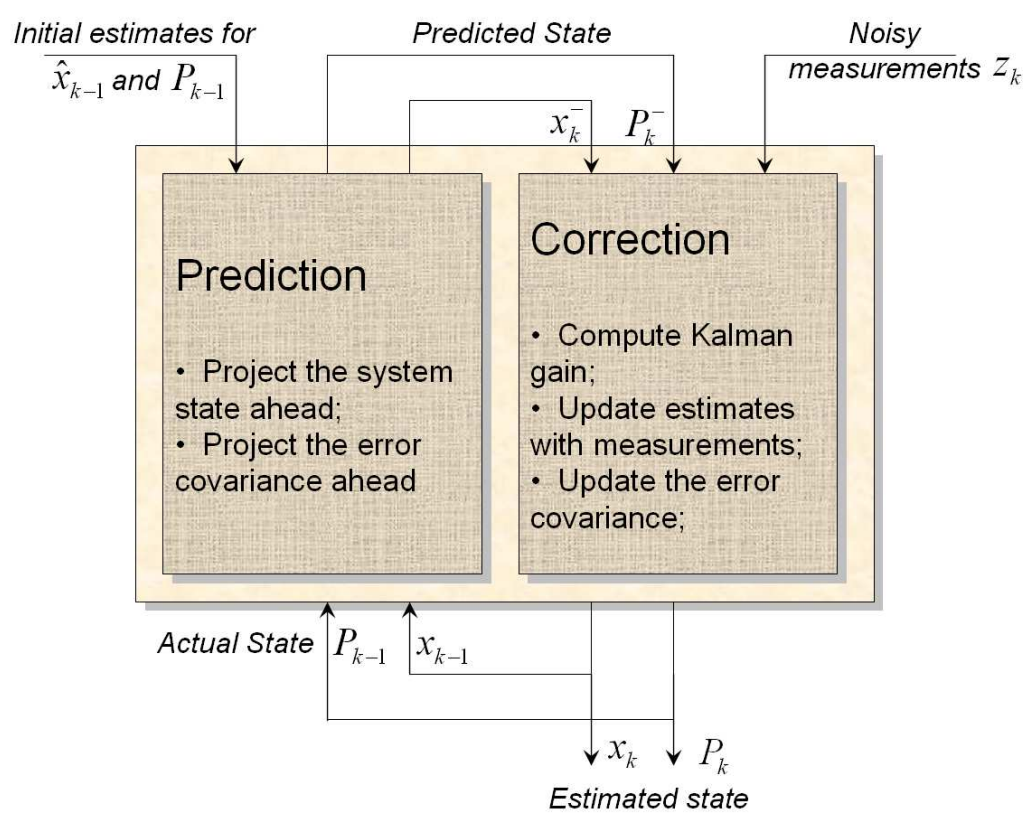

Figure 4. General prediction-correction structure of Kalman Filter.

A discrete-time process [4] with the state $x \in \Re^{n}$ is governed by the linear stochastic difference equation and has a measurement equation as follows:

$$
\begin{aligned}
& x_{k}=A x_{k-1}+B u_{k-1}+w_{k-1}, \\
& z_{k}=H x_{k}+v_{k} .
\end{aligned}
$$

Here $z \in \Re^{m}, w_{k}$ and $v_{k}$ representing process and measurement noises at time instance $k: p(w) \sim N(0, Q)$ and $p(v) \sim N(0, R)$. Then the discrete Kalman filter consists of 2 stages [4]: prediction or time update:

$$
\begin{aligned}
& \hat{x}_{k}^{-}=A \hat{x}_{k-1}+B u_{k-1}, \\
& P_{k}^{-}=A P_{k-1} A^{T}+Q,
\end{aligned}
$$

and correction or measurement update:

$$
\begin{aligned}
& K_{k}=P_{k}^{-} H^{T}\left(H P_{k}^{-} H^{T}+R\right)^{-1}, \\
& \hat{x}_{k}=\hat{x}_{k}^{-}+K_{k}\left(z_{k}-H \hat{x}_{k}^{-}\right), \\
& P_{k}=\left(I-K_{k} H\right) P_{k}^{-} .
\end{aligned}
$$


Here $A, B, H$ are from the description of the system, $P_{k}^{-}$and $\hat{x}_{k}^{-}$are the a priori estimate of error covariance and the state estimate, $P_{k}$ and $\hat{x}_{k}$ are the corresponding posteriori estimates and $K_{k}$ is the Kalman gain [1].

The Position-based Kalman Filter (P-KF) is designed with only the position variable in the state. The Position-Velocity Kalman filter (PV-KF) is derived from the P-KF by adding the $1^{\text {st }}$ order derivative (velocity) to the estimated state. The premise regarding a time-invariant model can be easily violated by a human hand motion, where several regimes do exist depending on the user activity. Single fixed-model Kalman filter can fail to cover all possible situations of interest. In practice this issue can be resolved by using adaptive Kalman filter schemes such as, for example, Interacting Multiple-Model (IMM$\mathrm{KF}$ ) methods, where a finite number of the process models is running in parallel and a soft switching scheme is used for state estimation from the combination of models based on calculated likelihoods (see [1] for details on IMM-KF).

\section{Methods}

Consider a Position Fractional Kalman Filter of the order $\alpha(\alpha \mathrm{P}-\mathrm{FKF})$ as a generalization of the linear Kalman filter for position estimation. Recall (2.1) and with the assumption for the process model:

$$
{ }_{(t-L)} D_{t}^{\alpha}[x(t)] \approx_{(t-L+h)} D_{t+h}^{\alpha}[x(t)],
$$

for $h=1, N(t)=L / h=N$, we obtain:

$$
\begin{aligned}
& \Delta^{\alpha} x_{k-1} \approx \sum_{j=0}^{N} b_{j} x_{k-1-j}, \\
& \Delta^{\alpha} x_{k} \approx \sum_{i=0}^{N+1} b_{i} x_{k-i}=b_{0} x_{k}+\sum_{i=1}^{N+1} b_{i} x_{k-i} .
\end{aligned}
$$

By assuming $\Delta^{\alpha} x_{k} \approx \Delta^{\alpha} x_{k-1}$ it follows:

$$
x_{k}=\frac{1}{b_{0}}\left(\sum_{j=0}^{N} b_{j} x_{k-1-j}-\sum_{i=1}^{N+1} b_{i} x_{k-i}\right)
$$

with $b_{0}=1$. With $d_{i}=b_{i}-b_{i+1}$ the state-space representation is given as

$$
\left[\begin{array}{c}
x_{k} \\
x_{k-1} \\
\vdots \\
x_{k-N+1} \\
x_{k-N}
\end{array}\right] \approx\left[\begin{array}{ccccc}
d_{0} & d_{1} & \cdots & d_{N-1} & d_{N} \\
1 & 0 & \cdots & 0 & 0 \\
0 & 1 & \cdots & 0 & 0 \\
\vdots & \vdots & \ddots & \vdots & \vdots \\
0 & 0 & \cdots & 1 & 0
\end{array}\right]\left[\begin{array}{c}
x_{k-1} \\
x_{k-2} \\
\vdots \\
x_{k-N} \\
x_{k-N-1}
\end{array}\right]
$$

It can be easily shown that IO derivatives in the form of the finite differences can be obtained as special cases from (3.1). The parameter $h$ cancels out from the expression above, although it obviously scales the noise matrices of the 
process. For the design of the KF we assume the completeness of the state, take $\Psi_{i}=\Upsilon_{i-1}-\Upsilon_{i}$ and for non-zero input:

$$
x_{k}^{-}=\sum_{i=1}^{N+1} \Psi_{i} x_{k-i}+B u_{k-1} .
$$

Here

$$
\Upsilon_{i}=\operatorname{diag}\left[(-1)^{i}\left(\begin{array}{c}
\alpha_{1} \\
i
\end{array}\right) \text { ‥ } \quad(-1)^{i}\left(\begin{array}{c}
\alpha_{M} \\
i
\end{array}\right)\right],
$$

the orders of the $M$ system equations are $\alpha_{1}, \ldots, \alpha_{M}$ and $x_{0}$ and $P_{0}$ are initial conditions. The obtained result is similar to the one in [17]. $\alpha \mathrm{P}-\mathrm{FKF}$ allows a direct substitution of $\alpha=1$, but it doesn't permit to set the $Q$ values separately for the derivative estimation. Proceeding similarly as for the case of $\alpha \mathrm{P}-\mathrm{FKF}$ :

$$
\Delta^{\alpha} x_{k-1} \approx \sum_{j=0}^{N} b_{j} x_{k-1-j}, \quad \Delta^{\alpha} x_{k} \approx \sum_{j=0}^{N+1} b_{j} x_{k-j} .
$$

Assuming $\Delta^{\alpha} x_{k} \approx \Delta^{\alpha} x_{k-1}$ we get:

$$
x_{k} \approx \Delta^{\alpha} x_{k-1}-\sum_{j=1}^{N+1} b_{j} x_{k-j}, \quad \Delta^{\alpha} x_{k} \approx \sum_{j=0}^{N} b_{j} x_{k-1-j} .
$$

Augmenting the state with the FD and previous state estimates for zero input we obtain

$$
\left[\begin{array}{c}
x_{k} \\
x_{k-1} \\
\vdots \\
x_{k-N} \\
\Delta^{\alpha} x_{k} \\
\Delta^{\alpha} x_{k-1} \\
\vdots \\
\Delta^{\alpha} x_{k-N}
\end{array}\right] \approx\left[\begin{array}{cccccccc}
-b_{1} & -b_{2} & \cdots & -b_{N+1} & h^{n} & 0 & \cdots & 0 \\
1 & 0 & \cdots & 0 & 0 & 0 & \cdots & 0 \\
\vdots & \vdots & \ddots & \vdots & \vdots & \vdots & \ddots & \vdots \\
0 & 0 & \cdots & 0 & 0 & 0 & \cdots & 0 \\
0 & 0 & \cdots & 0 & 1 & 0 & \cdots & 0 \\
0 & 0 & \cdots & 0 & 0 & 1 & \cdots & 0 \\
\vdots & \vdots & \ddots & \vdots & \vdots & \vdots & \ddots & \vdots \\
0 & 0 & \cdots & 0 & 0 & 0 & \cdots & 1
\end{array}\right]\left[\begin{array}{c}
x_{k-1} \\
x_{k-2} \\
\vdots \\
x_{k-N-1} \\
\Delta^{\alpha} x_{k-1} \\
\Delta^{\alpha} x_{k-2} \\
\vdots \\
\Delta^{\alpha} x_{k-N-1}
\end{array}\right]
$$

Here $h$ is the discretization step from the definition of the FD. This is equivalent to the IO difference schemes for $\alpha \in \mathbb{Z}$. For example with $n=1$ :

$$
x_{k}=x_{k-1}+h^{\alpha} \Delta^{\alpha} x_{k-1}=x_{k-1}+\Delta T v .
$$

Here $\Delta T$ is a time interval and $v$ is the velocity. Following (3.2),(3.3) and (3.4) we can rewrite the prediction step and generalize it for the system equations $M>1$ :

$$
\begin{aligned}
& \Phi_{1}=\left[\begin{array}{cc}
-\Upsilon_{1} & I \\
\Upsilon_{0} & 0
\end{array}\right], \quad \Phi_{i}=\left[\begin{array}{cc}
-\Upsilon_{i} & 0 \\
\Upsilon_{i-1} & 0
\end{array}\right], \quad i>1, \\
& {\left[\begin{array}{c}
x_{k} \\
\Delta^{\alpha} x_{k}
\end{array}\right]^{-}=\sum_{j=1}^{N+1} \Phi_{j}\left[\begin{array}{c}
x_{k-j} \\
\Delta^{\alpha} x_{k-j}
\end{array}\right]+B u_{k-1},}
\end{aligned}
$$


with $\Delta^{0} x_{k}=x_{k}$. For both $\alpha \mathrm{P}-\mathrm{FKF}$ and $\alpha \mathrm{PV}$-FKF the rest of the equations are identical to $(2.2)-(2.4)$ and the difference from the standard $\mathrm{KF}$ is in the prediction step only. We don't update the history estimates in the prediction step, thus the process noise is added only to $x_{k}$ ( $\left.\alpha \mathrm{P}-\mathrm{FKF}\right)$ or to both $x_{k}$ and $\Delta^{\alpha} x_{k}$ ( $\alpha$ PV-FKF). Differently from [17], $P_{k}^{-}$can't be simplified similarly to state prediction due to correlation introduced by the state augmentation and we estimate FD together with the state variable. Filters can be constructed either component-wise (separate filters for $X, Y$ and $Z$ ) or by combining them within the state if any coupling is of interest. Upper summation limit has to be changed to the last $N$ samples and sampling period $T_{s}$ considered.

\section{Experimental Results}

The choice of a process model for sensor fusion algorithm is crucial for achieving good performance at a low sensor measurement rate. In practice a fixed-order model is considered as a trade-off between noise and delay, while adaptive filtering schemes can be used to handle the varying dynamics of the system. In order to imitate a linear filtering case for a sensor fusion problem, we evaluated the performance of the developed methods on the position measurements (correspondingly $x, y$ and $z$ object position components) obtained with the help of Vicon ${ }^{T M}$ Motion Tracking System. For this purpose we used human head (HEAD) and hand (HAND) motion data sets recorded with a sampling period $T_{s}=0.02 \mathrm{sec}$ (further we take $T_{s}=1 \mathrm{sec}$. for simplicity). The recorded data represent typical user activity in virtual-reality applications. Noisy measurements were generated by adding Gaussian noise with $\sigma=0.05 \mathrm{~m}$ to each position component of the $\operatorname{Vicon}^{T M}$ data.

For a comparative analysis with IO process models we evaluated the performance of a conventional position Kalman filter (P-KF) and a position-velocity Kalman filter (PV-KF) on the same data sets. The conventional P-KF is a special case of (3.1) with position variables inside the state vector, $\alpha=0$ and no history augmentation. Similarly, the conventional PV-KF is a special case of (3.4) with positions and velocities forming the state vector, $\alpha=1$ and no history augmentation. Basic P-KF and PV-KF were compared to our proposed fractional extensions as shown in (3.1) and (3.4): $\alpha \mathrm{P}-\mathrm{FKF}$ and $\alpha \mathrm{PV}-\mathrm{FKF}$. The fractionality parameter $\alpha$ was allowed to be different for each component in $\alpha_{i} \mathrm{PV}-\mathrm{FKF}$ and is fixed to be the same for all 3 components in $\alpha \mathrm{P}-\mathrm{FKF}$ and $\alpha \mathrm{PV}$-FKF. We also compared the performance of our filters to basic adaptive filtering scheme, namely IMM-KF, commonly used in tracking applications. The IMM-KF was designed for switching between 2 models equivalent to standard P-KF and PV-KF as described above [1].

For all filters the measurement noise was taken to be known with the true value $R=0.0025 I_{n}$ and $P_{0}=10 I_{n}$ for all filters except IMM-KF. RMS error metric $\left(e_{r m s}\right)$ was used as a criteria for filter performance evaluation, whereas the maximum deviation of the filtered signal from the noiseless reference $\left(e_{\infty}\right)$ is presented for examination purpose only. We have used history length of $N=20$ samples since the experiments showed it to be enough for our purpose. The best set for the rest of parameters was found using Matlab ${ }^{T M}$ fminsearch routine 
and corresponding numerical values are presented in Table 1 and Table 2.

Table 1. Performance of different Kalman Filters for the HEAD data set.

\begin{tabular}{|c|c|c|c|}
\hline Filter Type & $e_{r m s}$ & $e_{\infty}$ & Parameters \\
\hline No Filter & 0.0878 & 0.2534 & \\
\hline $\mathrm{P}-\mathrm{KF}$ & 0.0422 & 0.1215 & $Q=2.8 \cdot 10^{-5}$ \\
\hline$\alpha \mathrm{P}-\mathrm{FKF}$ & 0.0370 & 0.1098 & $\alpha=0.95, Q=1.17 \cdot 10^{-7}$ \\
\hline $\mathrm{PV}-\mathrm{KF}$ & 0.0383 & 0.1044 & $Q_{p}=1.0 \cdot 10^{-5}, Q_{p v}=1.0 \cdot 10^{-7}$ \\
\hline$\alpha \mathrm{PV}-\mathrm{FKF}$ & 0.0365 & 0.1040 & $\begin{array}{l}\alpha=0.75, Q_{p}=1.82 \cdot 10^{-7} \\
Q_{p v}=3.27 \cdot 10^{-7}\end{array}$ \\
\hline$\alpha_{i}$ PV-FKF & 0.0356 & 0.1255 & $\begin{array}{l}\alpha_{x}=0.69, \alpha_{y}=0.70, \alpha_{z}=0.42 \\
Q_{p}=1.19 \cdot 10^{-9}, Q_{p v}=5.94 \cdot 10^{-7}\end{array}$ \\
\hline IMM KF & 0.0336 & 0.1499 & $\begin{array}{l}Q_{p}^{P}=4.89 \cdot 10^{-8}, Q_{p}^{P V}=1 \cdot 10^{-8} \\
Q_{p v}^{P V}=3.5610^{-7}, p_{12}=\frac{1}{20}, P_{0}=I_{n}\end{array}$ \\
\hline
\end{tabular}

Table 2. Performance of different Kalman Filters for the HAND data set.

\begin{tabular}{|c|c|c|c|}
\hline Filter Type & $e_{r m s}$ & $e_{\infty}$ & Parameters \\
\hline No Filter & 0.0870 & 0.2493 & \\
\hline $\mathrm{P}-\mathrm{KF}$ & 0.0442 & 0.1303 & $Q=3.56 \cdot 10^{-5}$ \\
\hline$\alpha \mathrm{P}-\mathrm{FKF}$ & 0.0416 & 0.1540 & $\alpha=0.80, Q=6.73 \cdot 10^{-7}$ \\
\hline $\mathrm{PV}-\mathrm{KF}$ & 0.0418 & 0.1544 & $Q_{p}=1.0 \cdot 10^{-5}, Q_{p v}=1.0 \cdot 10^{-5}$ \\
\hline$\alpha \mathrm{PV}-\mathrm{FKF}$ & 0.0407 & 0.1541 & $\begin{array}{l}\alpha=0.54, Q_{p}=7.42 \cdot 10^{-7} \\
Q_{p v}=2.42 \cdot 10^{-6}\end{array}$ \\
\hline$\alpha_{i} \mathrm{PV}-\mathrm{FKF}$ & 0.0405 & 0.1541 & $\begin{array}{l}\alpha_{x}=0.51, \alpha_{y}=0.55, \alpha_{z}=0.43 \\
Q_{p}=3.09 \cdot 10^{-6}, Q_{p v}=2.06 \cdot 10^{-6}\end{array}$ \\
\hline IMM KF & 0.0391 & 0.1534 & $\begin{array}{l}Q_{p}^{P}=3.50 \cdot 10^{-7}, Q_{p}^{P V}=5.73 \cdot 10^{-6} \\
Q_{p v}^{P V}=1.210^{-6}, p_{12}=\frac{1}{20}, P_{0}=I_{n}\end{array}$ \\
\hline
\end{tabular}

The performance of $\alpha \mathrm{P}-\mathrm{FKF}$ for different values of fractionality $\alpha$ and process noise $Q$ is shown in Fig. 5a. The result confirms $e_{r m s}$ sensitivity to $\alpha$ to be related to our confidence with the process model in terms of $Q$. Although the filters of fixed $\alpha$ have the same drawback of poor fitting to varying dynamics, reasonable performance improvement over basic IO filters can be achieved (Table 1 and Table 2) for the best set of parameters. A small performance gain is found in $\alpha_{i} \mathrm{PV}-\mathrm{FKF}$ filters probably due to all 3 components of motion having similar dynamics over the measurement time (4 min.). For given data sets the best found component-wise fractionality $\alpha_{z}$ (vertical axis) is smaller than $\alpha_{x}$ and $\alpha_{y}$ and can be explained by the user motion. Although constant order FKF outperform conventional P-KF and PV-KF schemes, their performance is still inferior to that of IMM-KF due to the latter's adaptive nature. The prediction power of FO models compared to PV-KF can be seen in Fig. 5b, clearly showing correlation features introduced by FD.

\section{Conclusions}

The work presents the construction of FO linear Kalman filter using the GL approximation. Two different types ( $\alpha \mathrm{P}$-FKF and augmented state $\alpha \mathrm{PV}-\mathrm{FKF})$ 


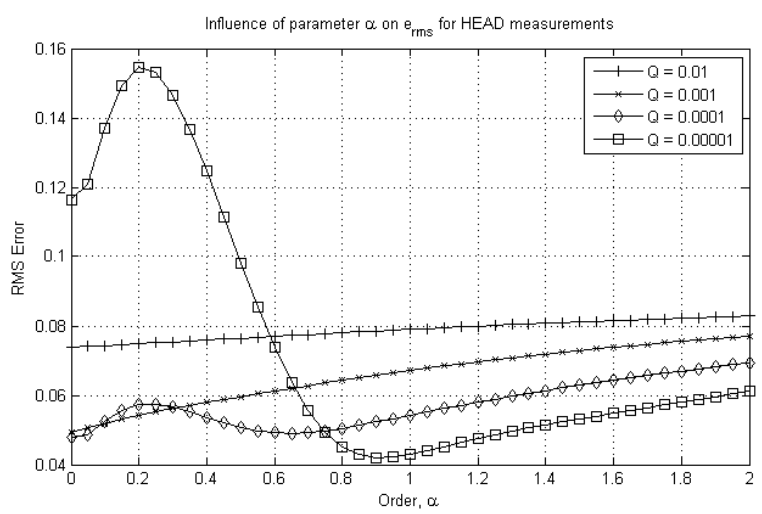

a)

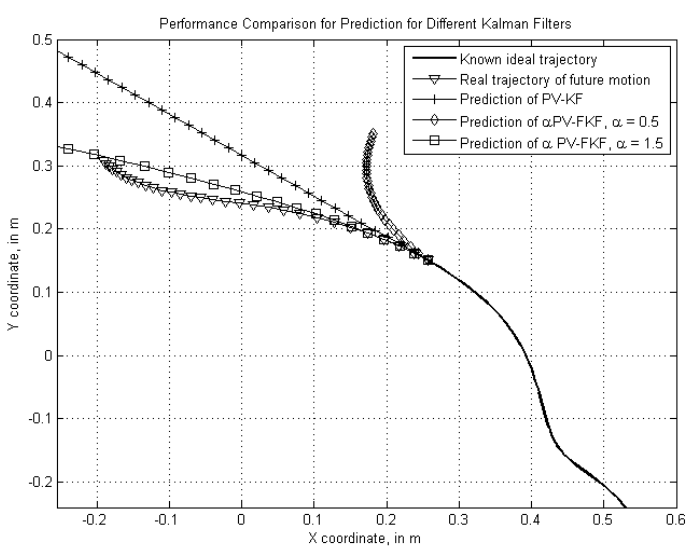

b)

Figure 5. a) Influence of the model order $\alpha$ on the performance of the $\alpha \mathrm{P}-\mathrm{FKF}$ for different values of process noise $Q$ : RMS error measure $e_{r m s}$ for HEAD data set. Similar results are obtained for HAND data set; b) Performance of PV-KF and $\alpha \mathrm{P}-\mathrm{FKF}$ in predicting real hand motion (straight line corresponds to PV-FKF).

of FKF have been implemented and compared to conventional KF implementations (P-KF, PV-KF, IMM-KF). The paper shows feasibility of such a construction for real-life limb tracking application. FD-based filters showed improved performance compared to fixed IO versions, although being slightly inferior to the adaptive scheme of an IMM-KF. Further research is required for efficient approximations, nonlinear models, adaptive fractional filtering schemes and applicability of FC as a solution for advanced noise models in fusion algorithms.

\section{References}

[1] Y. Bar-Shalom, X. R. Li and T. Kirubarajan. Estimation with Applications to Tracking and Engineering: Theory, Algorithms and Software. John Wiley \& 
Sons, Inc. New York, 2001.

[2] Y. Chen, R. Sun and A. Zhou. An overview of fractional order signal processing (FOSP) techniques. In Proc. ASME 2007 International Design Engineering Technical Conferences, Computers and Information in Engineering Conference, pp. 1205-1222. ASME, 2007.

[3] K. Debnath. Recent applications of fractional calculus to science and engineering. Int. J. Math. Sci., 2003(54):3413-3442, 2003.

[4] M. S. Grewal and A. P. Andrews. Kalman Filtering. Theory and Practice using MATLAB, 2nd edition. John Wiley \& Sons, Inc. New York, 2001.

[5] J. A. Tenreiro Machado. A probabilistic approach of the fractional-order differentiation. J. Fract. Calc. \& A Appl. Anal., 6(1):73-80, 2003.

[6] K. S. Miller. Derivatives of noninteger Order. Math. Mag., 68(3):183-192, 1995.

[7] K. B. Oldham and J. Spanier. The Fractional Calculus. Academic Press, New York, 1974. Mathematics in Science and Engineering, vol. 111

[8] M. D. Ortigueira. Introduction to fractional linear systems. Part 1: continuoustime case. IEE Proc. Vis. Image Signal Process., 147(1):62-70, 2000.

[9] M. D. Ortigueira. Introduction to fractional linear systems. Part 2: discrete-time case. IEE Proc. Vis. Image Signal Process., 147(1):71-78, 2000.

[10] M. D. Ortigueira. A coherent approach to non-integer order derivatives. Signal Proces. Spec. Sec.: Fract. Calc. Appl. Signal Syst., 86(10):2505-2515, 2006.

[11] M. D. Ortigueira, J. A. Machado and J. S. da Costa. Which differintegration? IEE Proc. Vis. Image Signal Process., 152(6):846-850, 2005.

[12] I. Podlubny. Fractional Differential Equations. Academic Press, California, 1999. Mathematics in Science and Engineering, vol. 198

[13] I. Podlubny. Geometric and physical interpretation of fractional integration and fractional differentiation. J. Fract. Calc. \& Appl. Anal., 5(4):367-386, 2002.

[14] R. S. Rutman. On physical interpretations of fractional integration and differentiation. Theoretical and Mathematical Physics, 105(3):1509-1519, 1995.

[15] S. G. Samko, A. A. Kilbas and O. I. Marichev. Fractional integrals and derivatives - theory and applications. Gordon and Breach Science Publishers, 1987.

[16] M. Sekine, M. Akay, T. Tamura, Y. Higashi and T. Fujimoto. Fractal dynamics of body motion in patients with Parkinson's disease. J. Neural Eng., 1(1):8-15, 2004.

[17] D. Sierociuk and A. Dzielinski. Fractional Kalman filter algorithms for the states, parameters and order of fractional system estimation. Int. J. Appl. Math. Comput. Sci, 16(1):129-140, 2006. 